\title{
Neurofeedback na Reabilitaçáo Neuropsicológica Pós-Acidente Vascular Cerebral
}

\author{
Neurofeedback In Neuropsychological Rehabilitation After Stroke
}

Maria Flávia Porcaro Muratori ${ }^{1}$, Tânia Maria Porcaro Muratori ${ }^{2}$

\section{RESUMO}

Introduçáo. Embora ainda subutilizado no Brasil, o neurofeedback constitui uma ferramenta clínica amplamente utilizada nos EUA e na Europa. Este estudo teve como objetivo analisar os efeitos do neurofeedback na reabilitação neuropsicológica de pacientes pós-Acidente Vascular Cerebral (AVC). Método. Revisão de literatura com publicações de 1965 a 2008. Foram incluídas publicaçôes que abordavam os efeitos do neurofeedback como recurso terapêutico, especialmente, na reabilitação cognitiva e psicológica pós-AVC. Resultados. Neurofeedback ou também chamado eletroencefalograma (EEG) biofeedback proporciona intervenção direta sobre o cérebro, de maneira não invasiva, obtendo a informação elétrica do cérebro e permitindo que o paciente atue sobre esta de forma consciente. A literatura revisada aponta para a adoçáo do neurofeedback como uma promissora ferramenta para o gerenciamento de várias disfunçóes cognitivas e emocionais pós-AVC, a qual parece fornecer benefícios fora do intervalo de tempo em que é esperada uma melhora espontânea. Conclusáo. O campo do neurofeedback está se configurando como uma grande promessa para a reabilitação da atenção, processamento da linguagem e memória de trabalho. Os relatórios de estudos de caso têm sido encorajadores, porém mais pesquisas são necessárias para determinar a eficácia desta abordagem em relação ao quadro clínico e em relação à sua combinação com outras abordagens de reabilitação cognitiva pósAVC.

Unitermos. Acidente Vascular Cerebral, Neurorretroalimentação, Biorretroalimentação, Reabilitação.

Citaçáo. Muratori MFP, Muratori TMP. Neurofeedback na Reabilitação Neuropsicológica Pós-Acidente Vascular Cerebral.

\begin{abstract}
Introduction. While still underused in Brazil, neurofeedback is a clinical tool used in the U.S. and in Europe. This study aimed to examine the effects of neurofeedback in the neuropsychological rehabilitation of patients after stroke. Method. Literature review on publications from 1965 to 2008. Publications were included that addressed the effects of neurofeedback as a therapeutic resource, especially in cognitive and psychological rehabilitation after stroke. Results. Neurofeedback or also called electroencephalography (EEG) biofeedback provides direct intervention on the brain, noninvasively, obtaining electrical information from the brain and allowing the patient to act on this consciously. The literature reviewed points to the adoption of neurofeedback as a promising tool for managing various cognitive and emotional dysfunctions after stroke, which appears to provide benefits beyond the time interval in which spontaneous improvement is expected. Conclusion. The neurofeedback is shaping up as a great promise for the rehabilitation of attention, language processing and working memory. Reports of case studies have been encouraging but more research is needed to determine the effectiveness of this approach in relation to the clinical characteristic and in relation to its combination with other approaches to cognitive rehabilitation after stroke.
\end{abstract}

Keywords. Stroke, Neurofeedback, Biofeedback, Rehabilitation.

Citation. Muratori MFP, Muratori TMP. Neurofeedback In Neuropsychological Rehabilitation After Stroke.

\footnotetext{
Trabalho realizado na Pontifícia Universidade Católica do Paraná (PUCPR), Curitiba-PR, Brasil.

1.Psicóloga Clínica, São João Del Rei - MG, Brasil. Graduada em odontologia pela UFJF (Universidade Federal de Juiz de Fora); Graduada em Psicologia pela UFSJ (Universidade Federal de São João Del Rei); Pós-graduada em Psicologia Transpessoal pela Faculdade Metropolitana de Belo Horizonte - MG; Formação em Biofeedback / Neurofeedback pela The Learning Curve, Inc.

2.Psicóloga Clínica, Curitiba - PR, Brasil. Graduada em odontologia pela UFJF (Universidade Federal de Juiz de Fora); Graduada em Psicologia pela UFJF; Pós-graduada em Psicologia Transpessoal pelas Faculdades Integradas "Espírita" (UNIBEM) de Curitiba-PR; Pós-graduanda em Neurociência pela PUC-PR; Formaçáo em Biofeedback / Neurofeedback pela The Learning Curve, Inc.
}

Endereço para correspondência: Tânia Maria Porcaro Muratori Rua Nilo Peçanha, 2.222/casa17, Bairro São Lourenço CEP 82120-440, Curitiba-PR, Brasil. E-mail: taniamariapsi@hotmail.com 


\section{INTRODUÇÃO}

Um acidente vascular cerebral (AVC) distingui-se pela instalação de um déficit neurológico focal repentino, decorrente de uma lesão cerebral, originada por um distúrbio vascular e não traumático. Podem ocorrer AVCs isquêmicos ou hemorrágicos. Como resultado, dependendo do local e da extensão da área afetada, as manifestaçôes clínicas podem incluir alteraçôes funcionais motoras, sensitivas, cognitivas, perceptivas e da linguagem ${ }^{1}$.

Os idosos são a maior parte afetada, mas existe uma percentagem de $20 \%$ dos AVCs, que ocorre em indivíduos abaixo dos 65 anos. O tratamento para AVC pode incluir medicação, cirurgia e reabilitação, sendo que esta última pode envolver terapias fonoaudiológicas, fisioterapêuticas, terapêuticas ocupacionais e psicoterapêuticas ${ }^{1}$.

A incidência do AVC é de aproximadamente 158 por 100.000 e é uma das principais causas de incapacidade nos Estados Unidos ${ }^{2,3}$. Há cerca de 4,4 milhóes de sobreviventes de AVC nos Estados Unidos, e quase 40\% desses indivíduos apresentam, pelo menos, uma moderada limitação funcional, e entre $15 \%$ e $30 \%$ apresentam deficiências graves ${ }^{4}$.

Sequelas físicas comuns incluem hemiparesia ou hemiplegia, incontinência urinária, bem como disfagia e disartria 5 . Sequelas cognitivas comuns ao AVC incluem afasia, abulia, o comprometimento da atenção e memória, falta de percepção espacial, mudanças de personalidade e anosognosia ${ }^{2,6,7}$.

Os esforços de reabilitação podem oferecer um efeito positivo significativo sobre o grau de deficiência que indivíduos experimentam nas semanas e meses pós$\mathrm{AVC}^{8}$. Algumas revisóes sistemáticas da literatura têm revelado benefícios específicos e resultados funcionais significativos com fisioterapia ${ }^{9}$, terapia ocupacional ${ }^{10}$ e reabilitação cognitiva ${ }^{11}$.

É importante mencionar que cerca de $40 \%$ dos sobreviventes de AVC apresentam déficits cognitivos. Após a fase aguda, a reabilitação cognitiva tem um papel fundamental. Embora existam muitos tipos diferentes de treinamentos cognitivos potenciais, existem achados divergentes sobre a eficácia de tais intervenções ${ }^{12}$.

Programas de reabilitação cognitiva tradicionais nem sempre levam a uma melhora satisfatória. Recentes estudos a respeito da reabilitação pós-AVC apontam para a adoção do neurofeedback (neurorretroalimentação) como uma nova e promissora ferramenta para o gerenciamento de várias condições pós-AVC ${ }^{13}$.

Pesquisas sobre a plasticidade cerebral deram uma nova esperança para sobreviventes de acidente vascular cerebral. Embora os sistemas cerebrais de adultos tiveram sido pensados, historicamente, como estáticos, tem havido fortes evidências de que a anatomia e fisiologia do cérebro são plásticas, sendo possível a alteração funcional dos sistemas através da terapia com neurofeedback ${ }^{14}$.

Nesta técnica, os eletrodos do eletroencefalograma (EEG) registram a atividade das ondas elétricas em determinados locais do couro cabeludo e monitores de computador fornecem informaçôes sobre a atividade do cérebro do paciente. A maioria dos sistemas de neurofeedback pode apresentar uma variedade de informaçóes para o terapeuta e para o paciente, tais como: a composição das frequências medidas em ciclos por segundo (delta 0,5-4 Hz, teta $4-8 \mathrm{~Hz}$, alfa $8-12 \mathrm{~Hz}$, beta $13-21 \mathrm{~Hz}$, beta alta $21-38 \mathrm{~Hz}$ e gama $38-60 \mathrm{~Hz}$ ), a amplitude (medida da magnitude de cada frequência, ou seja, medida da voltagem em micro volts de cada frequência), a coerência (o nível de comunicação elétrica entre dois ou mais locais do cérebro em uma ou mais bandas de frequência), ou qualquer outro valor derivado destas medidas. Então, estas informações, ofertadas pelo equipamento do neurofeedback, são utilizadas para, através do condicionamento operante, combinado com várias estratégias cognitivas ou tarefas funcionais, alterar a atividade do EEG do paciente na direção desejada ${ }^{15}$.

Dada a relação existente entre a amplitude de determinada frequência de onda em local específico do córtex e a taxa de metabolismo desta estrutura cortical ${ }^{16}$, o neurofeedback permite ao paciente atuar sobre a atividade elétrica neural e, assim, sobre o metabolismo cortical e, consequentemente, sobre a neuroplasticidade ${ }^{17,18}$. Devido a este acesso direto ao funcionamento neural, o neurofeedback pode ser usado para alterar ou acelerar os processos de reorganização funcional de determinadas áreas do córtex, e, assim, acelerar a recuperação, ou em alguns casos, até permitir a recuperação funcional, que não teria ocorrido de outra forma ${ }^{14}$.

Como o neurofeedback ainda é subutilizado no Brasil, apesar de ser uma ferramenta clínica, amplamen- 
te, utilizada nos EUA e na Europa, o mesmo precisa ser mais divulgado e estudado no Brasil, pois muito trabalho precisa ser feito por aqueles indivíduos que sobrevivem a uma lesão cerebral, porque muito pouco se encontra disponível para eles e suas famílias até o momento. No entanto, existe a chance de se recuperar o quanto lhes for possível da funcionalidade perdida, ao invés de serem considerados como sem chance de recuperação.

O presente artigo foi feito como uma revisão da literatura sobre a eficácia da aplicação do neurofeedback em aspectos cognitivos e psicológicos na recuperação pós-AVC. O neurofeedback é um treinamento, que proporciona intervenção direta sobre o cérebro, de maneira não invasiva, obtendo a informação elétrica do cérebro, amplificando-a e promovendo uma representação visual para o paciente, o qual pode atuar sobre esta atividade elétrica, direcionando-a para o padrão de frequências e amplitudes desejadas naquela área do cérebro.

\section{MÉTODO}

Este trabalho é uma revisão de literatura sobre a eficácia da aplicação do neurofeedback na reabilitação neuropsicológica pós-AVC. O levantamento das referências bibliográficas, para elaboração desta revisão, foi realizado através da base de dados PubMed/MEDLINE. A busca de artigos foi realizada no idioma inglês e foram utilizadas como palavras-chave: stroke, biofeedback, neurofeedback, rehabilitation. Foi realizada também uma busca manual nestas referências encontradas nas bases eletrônicas.

Após a extração das referências, avaliou-se a relevância, qualidade e especificidade dos estudos. Foram selecionados livros e artigos, escritos em inglês, publicados entre os anos de 1965 a 2008. Os critérios de inclusão foram: utilizar publicaçôes de estudos experimentais, que abordassem os efeitos do neurofeedback como recurso terapêutico na reabilitação psicológica ou cognitiva pósAVC; Utilizar revisóes de literatura abordando os conceitos e princípios básicos do neurofeedback; Priorizar estudos controlados, com número considerável de participantes e com avaliaçóes de desempenho a partir de instrumentos bem-fundamentados; Priorizar estudos indexados no PubMed (simplesmente pelo fato de que essa base permite livre acesso).
Os seis estudos com resultados mais relevantes para o campo foram apresentados na Tabela 1. Para a formação da tabela foram priorizados: 1 . Estudos controlados; 2. Estudos que apontem resultados positivos ou negativos; 3. Estudos com grupos ativos, preferencialmente, grandes; 4. Estudos com avaliação de desempenho bem fundamentada.

\section{RESULTADOS}

Inicialmente, a intenção era apenas incluir estudos controlados, com número considerável de participantes, com avaliaçóes de desempenho a partir de instrumentos bem-fundamentados e indexados no PubMed, contudo, devido às limitações da literatura, é necessário considerar que isto nem sempre é possível.

Foram encontrados 156 artigos no PubMed sob as palavras-chave Neurofeedback or Biofeedback. Porém, ao se fazer a filtragem dos artigos referentes à reabilitação psicológica ou cognitiva pós-AVC, este número foi, drasticamente, reduzido para 17.

A elaboração da tabela 1 foi dificultada devido ao fato da literatura apresentar estudos com um número muito reduzido de participantes e com controle deficiente. Sendo que a tabela apresenta dois estudos com apenas um indivíduo participante.

\section{DISCUSSÃO}

\section{Neurofeedback}

O termo biofeedback (biorretroalimentação) engloba várias modalidades técnicas, que fornecem informaçóes sobre processos fisiológicos corporais inconscientes com o propósito de torná-los conscientes para que possam ser manipulados. Os procedimentos utilizam equipamentos eletrônicos para monitorar os processos fisiológicos selecionados (por exemplo, a frequência cardíaca, a taxa respiratória, a temperatura da pele, a tensão muscular ou as ondas elétricas cerebrais) e, em seguida, converter estas medidas em respostas (feedback), através de sinais visuais e auditivos, que o paciente pode perceber facilmente ${ }^{19}$.

O neurofeedback (neurorretroalimentação) se refere a um tipo específico de biofeedback, que fornece informaçôes sobre as frequências e amplitudes das ondas elétricas cerebrais, através de eletrodos colocados em áre- 
as específicas do couro cabeludo, possibilitando que os participantes aprendam a regular voluntariamente as frequências das ondas cerebrais ${ }^{19}$.

As frequências das ondas elétricas cerebrais são medidas em ciclos por segundo ou hertz $(\mathrm{Hz})$, ou seja, a frequência é quantas vezes uma onda se repete dentro de um segundo. Sendo que diferentes taxas de frequências estão associadas a diferentes funções cerebrais. Ondas Gama (acima de $38 \mathrm{~Hz}$ ) estão envolvidas na maior atividade mental e consolidação das informações. Ondas beta $(13-21 \mathrm{~Hz})$ e beta alta $(21-38 \mathrm{~Hz})$ são ondas cerebrais rápidas associadas ao pensamento ativo, concentração, raciocínio lógico e processamento de informaçóes. As ondas alfa $(8-10 \mathrm{~Hz})$ e alfa alta $(10-12 \mathrm{~Hz})$ estão associadas com estados relaxados e calmos, descontração, atenção e consciência. Ondas Teta $(4-8 \mathrm{~Hz})$ estão associadas com relaxamento profundo, sonolência, criatividade e devaneios. As ondas delta são as mais lentas (abaixo de $4 \mathrm{~Hz}$ ) e estão associadas ao sono profundo ${ }^{15}$.

O primeiro passo, na intervenção com neurofeedback, é a execução de uma avaliação inicial, ou seja, a criação de um mapa funcional do cérebro do paciente, através de um EEG quantitativo (EEGQ), para determinar a extensão dos déficits e suas localizaçôes ${ }^{15}$. EEGQ é a eletroencefalografia quantitativa, mapeamento cerebral ou topografia da atividade elétrica cerebral. É uma técnica moderna de eletrofisiologia que permite uma abordagem funcional não invasiva do cérebro, por meio de uma representação topográfica quantificada da sua atividade elétrica.

O mapeamento cerebral fornece informaçóes sobre as várias ondas cerebrais analisadas num EEG, que são comparadas a uma normativa do banco de dados. Através das informaçóes do mapeamento do cérebro do paciente, são identificados os locais que necessitam de intervenção e as ondas cerebrais que precisam ser aumentadas ou diminuídas em suas amplitudes ${ }^{20}$.

A partir desta avaliação, um treinamento operante personalizado pode ser concebido pelo terapeuta, ou seja, traçam-se os protocolos de treinamento que serão aplicados neste paciente, centrando-se nas áreas de desvio, objetivando proporcionar-lhe um melhor desempenho de suas funções, através de alteraçôes no seu padrão elétrico. Os padrões elétricos do cérebro são modificá- veis através do condicionamento operante, sendo que as frequências cerebrais excessivas devem ser reduzidas e aquelas com déficit devem ser aumentadas ${ }^{20}$.

Durante uma sessão de treinamento com neurofeedback, o paciente se encontra sentado em uma cadeira confortável e os eletrodos são colocados em seu couro cabeludo em locais específicos de acordo com o sistema Internacional 10-20 de colocação de eletrodos (Figura 1). Os impulsos elétricos captados desses locais são exibidos em um monitor de computador na forma de gráficos ou outros meios visuais. O paciente, então, se envolve em uma atividade específica, recebendo, do programa do computador, sinalizaçôes contínuas de seu desempenho em tempo real ${ }^{20}$. Por exemplo, uma criança pode jogar um jogo de computador ou um adulto pode mover um gráfico de barras para cima ou para baixo, recebendo um feedback positivo (sinal sonoro e/ou uma mudança em um display visual) sempre que as ondas cerebrais estiverem na frequência e amplitude objetivadas.

O neurofeedback tem sido usado, eficazmente, para tratar vários distúrbios, incluindo a epilepsia ${ }^{15,21,22}$, o transtorno do déficit de atenção e hiperatividade $(\mathrm{TDAH})^{15,23-25}$, a enxaqueca ${ }^{26}$, os distúrbios do sono ${ }^{26}$, a síndrome da fadiga crônica/fibromialgia ${ }^{26}$, casos de dependência química ${ }^{15,27}$, transtorno desafiador opositivo e obsessivo-compulsivo $^{26}$ e casos de lesóes cerebrais ${ }^{28}$.

Em estudos realizados para medir a reabilitação da atenção, foram observados efeitos benéficos específicos, através do treinamento com o neurofeedback, inclusive em crianças e adultos com transtorno de déficit de atenção ${ }^{28}$. Estes estudos apontam que as dificuldades de atenção podem estar associadas, frequentemente, com a excessiva atividade de ondas lentas no EEG do córtex frontal em ambos os casos: déficit de atenção e após lesão cerebral $^{28}$.

Pesquisas recentes têm analisado o uso do neurofeedback para aumentar o desempenho atlético ${ }^{15,29}$ e a performance musical ${ }^{30}$. Há indicativos de efeitos positivos na cognição, na atenção e na memória após o treino com neurofeedback ${ }^{31}$. Além disso, alguns relatos de eficácia do neurofeedback na reabilitação após traumatismo craniano e acidente vascular cerebral estão registrados na literatura ${ }^{32,33}$. 


\begin{tabular}{|c|c|c|c|}
\hline Autor, data & $\begin{array}{l}\mathrm{n}^{\circ} \text { indivíduos } \\
\text { grupo ativo }\end{array}$ & Protocolo & Resultados Clínicos \\
\hline Ayers, $1981^{32}$ & 6 & $\begin{array}{c}\uparrow 12-15 \mathrm{~Hz} \\
\downarrow \text { Teta } \\
\downarrow \text { beta alta }\end{array}$ & $\uparrow$ Controle motor \\
\hline Ayers, $1987^{36}$ & 250 & $\downarrow$ Teta & $\begin{array}{c}\text { } \text { Energia e atenção } \\
\downarrow \text { Depressão, impulsividade, } \\
\text { sensibilidade a luz e ao som, } \\
\text { inversão de letras e palavras }\end{array}$ \\
\hline $\begin{array}{c}\text { Rozelle, } \\
\text { Budzynski, } \\
1995^{52}\end{array}$ & 1 & $\begin{array}{l}\downarrow \text { Teta } \\
\uparrow \text { Beta }\end{array}$ & $\begin{array}{l}\text { 个Fluência da fala, atenção, } \\
\text { concentração, coordenação } \\
\text { motora, equilíbrio, humor. }\end{array}$ \\
\hline Laibow et al, $2001^{53}$ & 27 & $\begin{array}{l}\downarrow \text { Teta } \\
\uparrow \text { Beta }\end{array}$ & $\uparrow$ Melhora dos sintomas \\
\hline Beardenet al, 20035 & 1 & $\downarrow$ Teta & $\begin{array}{l}\text { 个Atenção, memória, atenção } \\
\text { dividida, velocidade de proces- } \\
\text { samento e sequenciamento. }\end{array}$ \\
\hline $\begin{array}{l}\text { Doppelmayr et al, } \\
2007^{55} \\
\text { (experimento } 1 \text { ) }\end{array}$ & 15 & $\uparrow$ Alfa & 个Desempenho cognitivo \\
\hline \multirow{2}{*}{$\begin{array}{l}\text { Doppelmayr et al, } \\
2007^{55} \\
\text { (experimento 2) }\end{array}$} & 4 & $\downarrow$ Teta & 个Desempenho cognitivo \\
\hline & 7 & $\uparrow$ Alfa & 个Desempenho cognitivo \\
\hline
\end{tabular}

\section{Disfunçóes da Atividade Elétrica do Cérebro}

Existem quatro tipos de disfunçóes da atividade elétrica do cérebro (hipofunção, hiperfunção, desconexão e hiperconexão), que podem ser gerenciadas através do neurofeedback ${ }^{33-36}$. As etiologias mais comuns, relacionadas com estas disfunçốes são a congênita, o traumatismo craniano e o $\mathrm{AVC}^{35-39}$.

Lesões focais em determinadas áreas cerebrais resultam em hipofunção (redução da função) daquela área, comprometendo seu desempenho. Como algumas áreas cerebrais estão correlacionadas com funçôes cerebrais específicas, uma lesão da área de Paul Pierre Broca (18241880) (aproximadamente F7 no sistema 10/20, Figura 1) resultaria em uma disfunção expressiva da fala ${ }^{35}$. Uma lesão na área de Carl Wernicke (1848-1905) (aproximadamente T5 no sistema 10/20, Figura 1) resultaria em uma perda da compreensão da linguagem ${ }^{35}$.

Os protocolos de neurofeedback, utilizados no tratamento das hipofunçóes, objetivam aumentar a atividade elétrica em áreas específicas, diminuindo a amplitude das ondas lentas e aumentando a amplitude das ondas acima de $10 \mathrm{~Hz}^{35}$.
A hiperfunção está relacionada com a produção excessiva de frequências de atividade beta alta $(21-38 \mathrm{~Hz})$ em determinada região do cérebro. Esta disfunção está associada à ansiedade, irritabilidade e redução da tolerância ao estresse. Para corrigi-la, o neurofeedback promoveria a inibição de ondas de $21-38 \mathrm{~Hz}$ (beta alta) e o aumento da atividade de $10 \mathrm{~Hz}$ (alfa) ${ }^{35}$.

A desconexão ou hipocoerência é a falta de comunicação ou ligação entre áreas do cérebro, que precisam se conectar para a realização de tarefas, desencadeando alguns sintomas clínicos ${ }^{37,38}$. Por exemplo, a afasia resultante de um acidente vascular cerebral, que lesionou as vias de associação entre a área de Broca e a área de Wernicke.

Duas áreas cerebrais não conseguem se conectar quando as suas ondas cerebrais não trabalham em coerência. As desconexôes são comuns e numerosas em vários distúrbios clínicos ${ }^{35}$, como a dificuldade congênita de aprendizagem, traumatismo craniano $^{39}$, dislexia ${ }^{40}$, a epilepsia $^{41}$ e o autismo ${ }^{42}$. O treinamento com neurofeedback, para aumentar a coerência elétrica entre duas regiōes, pode restabelecer a conexão ${ }^{35}$.

A hiperconexão ou hipercoerência pode surgir da 


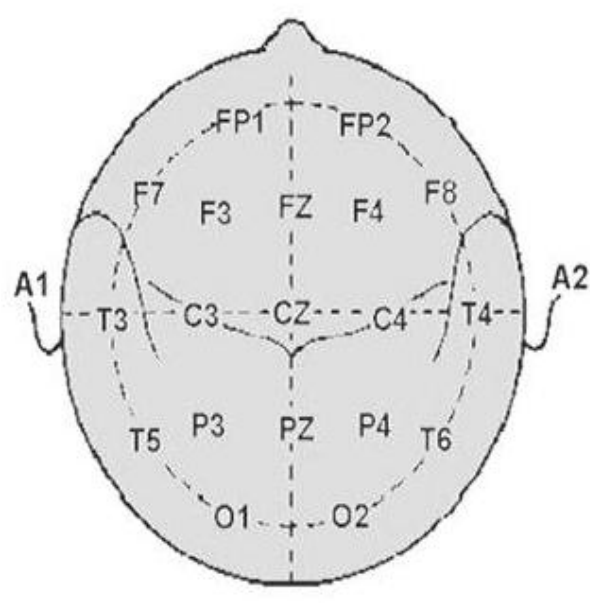

Figura 1. O sistema internacional 10-20 de colocação de eletrodos. (Adaptado de Rowan, Tolunsky, 200358).

falta de poda ou de conexóes extras ocorridas após uma lesão do cérebro, podendo ocorrer uma hiperconectividade em uma área específica e rupturas de conexôes desta área com outras regióes do cérebro. Um exemplo seria a combinação da hiperconectividade frontofrontal com a desconexão frontal com outras regióes do cérebro no autismo ${ }^{43}$.

\section{Neurofeedback na Reabilitaçáo Pós-Avc}

Alguns estudos demonstram a eficácia da técnica do neurofeedback, sua potencial capacidade de atuar sobre o cérebro e trazer benefícios em casos de lesôes cerebrais $^{32,33,36,44-48}$. Quando há uma lesão cerebral, uma das alteraçóes percebidas no EEGQ é um aumento da amplitude das frequências mais baixas, o cérebro fica mais lento. $\mathrm{O}$ treinamento consiste em reduzir a amplitude das ondas lentas, o que resulta em um aumento em funcionalidade ${ }^{36}$.

Foi investigado o uso do neurofeedback com a finalidade de aumentar a atividade das ondas alfa para melhorar o desempenho cognitivo, baseando-se na existente relação entre a amplitude das ondas alfa e das ondas teta com a funcionalidade cognitiva ${ }^{49,50}$. Faixas mais baixas de alfa têm sido associadas a distúrbios de atenção e grande amplitude na faixa teta tem sido associada com disfunção na memória de trabalho. Os resultados mostraram melhor desempenho em uma tarefa mental se o indivíduo fosse capaz de aumentar a potência em alfa através de neurofeedback ${ }^{50}$.
Em um estudo foram investigados seis pacientes com, pelo menos, três anos de pós-AVC ${ }^{32}$ (Tabela 1). Estes pacientes receberam eletromiográfico (EMG) biofeedback por dez meses para facilitar a extensão dos dedos. Em seguida, receberam EMG biofeedback combinado com neurofeedback no córtex sensório-motor esquerdo, para estimular a atividade na faixa de $12-15 \mathrm{~Hz}$ e inibir a atividade de $4-8 \mathrm{~Hz}$ (teta) e $21-38 \mathrm{~Hz}$ (beta alta) por mais cinco meses. Nenhum dos pacientes conseguiu alcançar a extensão dos dedos apenas com o EMG biofeedback, contudo quando o neurofeedback foi adicionado ao tratamento, eles alcançaram o controle da extensão dos $\operatorname{dedos}^{32}$.

Foram investigados, em uma pesquisa, duzentos e cinquenta pacientes com traumatismo craniano fechado ${ }^{36}$ (Tabela 1). Eles receberam vinte e quatro sessóes de neurofeedback destinadas a inibir as ondas teta $(4-7 \mathrm{~Hz})^{36}$. Todos estes pacientes relataram, após o tratamento, uma melhora significativa na energia e na atenção, bem como uma diminuição da depressão e da impulsividade. Além disso, esses pacientes experimentaram uma diminuição da sensibilidade à luz e ao som, bem como uma diminuição na inversão das letras e palavras ${ }^{51}$.

Um dos estudos de caso, que aparece na literatura, descreve o tratamento com neurofeedback em um paciente do sexo masculino de 55 anos de idade, que sofreu, um ano antes, um AVC isquêmico no hemisfério esquerdo, com infartos secundários temporo/parietal ${ }^{52}$ (Tabela 1). Antes do tratamento, o paciente relatou dificuldades em encontrar palavras, discurso interrompido, parafasia, e dificuldade em focar seu olho direito. A análise quantitativa do EEG revelou, no córtex esquerdo, um aumento de atividade 4-7 $\mathrm{Hz}$ e persistência de alta amplitude de ondas alfa com olhos abertos ${ }^{52}$.

Este paciente recebeu seis meses de treinamento com neurofeedback para inibir a atividade de 4-7 Hz (teta) e aumentar atividade de $15-21 \mathrm{~Hz}$ (beta) na região sensório-motora e nas áreas da fala no lado esquerdo do córtex. Em avaliação do paciente após o tratamento, o EEG quantitativo revelou a diminuição significativa da atividade das ondas lentas. Os autores relataram que o paciente também demonstrou melhoras significativas na fluência da fala, na atenção, na concentração, na coordenação motora, no equilíbrio e também no humor ${ }^{52}$. 
Contudo, uma vez que não foram utilizadas estatísticas formais neste estudo de caso, as melhorias relatadas não podem ser quantificadas objetivamente.

Como a recuperação espontânea, após um Acidente Vascular Cerebral, ocorre em sua maior parte dentro do primeiro ano, é importante notar que este paciente começou o neurofeedback cerca de um ano pós-AVC. Assim, esta experiência sugere que o neurofeedback, quando aplicado na recuperação do AVC, possibilita benefícios na reabilitação para além do período esperado de recuperação espontânea ${ }^{52}$.

Em um estudo foi verificada a eficiência do condicionamento operante, através do neurofeedback em um grupo experimental de vinte e sete pacientes ${ }^{53}$ (Tabela 1). Foi realizado o treinamento com um protocolo que visava diminuir a razão entre a atividade teta $(4-8 \mathrm{~Hz})$ e a atividade beta $(15-30 \mathrm{~Hz})$, ou seja, diminuir teta e aumentar beta $^{53}$. Isto com a finalidade de reduzir a gravidade dos sintomas gerais em indivíduos com lesão cerebral moderada a severa, decorrentes de lesão cerebral traumática, AVC ou hemisferectomia ${ }^{53}$. Apesar deste estudo ter incluído pacientes com lesão cerebral de outras etiologias, além das resultantes de AVC, ele aponta a utilidade potencial do neurofeedback nesta população de pacientes.

Outro trabalho descreve o tratamento de um paciente do sexo masculino de 52 anos de idade, um ano após os eventos cérebro vasculares, infartos no tálamo esquerdo e área temporoparietal do córtex ${ }^{54}$ (Tabela 1). Após quatorze sessōes de neurofeedback, para reduzir a atividade de ondas lentas nas áreas mais afetadas, ele demonstrou reduçóes significativas na atividade de 4-7 Hz no hemisfério esquerdo e atenuação da amplitude de alfa ao abrir os olhos. Ele também demonstrou melhorias estatisticamente significativas nos testes neuropsicológicos de atenção, memória de trabalho, atenção dividida, velocidade de processamento e sequenciamento ${ }^{54}$. Como o relatório deste estudo de caso, reporta a aplicaçáo deste método de tratamento em apenas um único paciente, seus resultados não podem ser quantificados estatisticamente, porém são encorajadores para novas pesquisas.

Para testar a hipótese de que diferentes tipos de neurofeedback contribuem para aumentar o desempenho cognitivo pós-AVC, dois experimentos foram realizados no Departamento de Neuropsicologia da Clínica de Re- abilitação Medical Park Loipl, na Alemanha ${ }^{55}$ (Tabela 1). Todos os participantes foram exaustivamente informados, participaram voluntariamente, assinaram um termo de consentimento e receberam o tratamento de reabilitação padrão utilizado nesta clínica, além dos procedimentos que foram projetados especificamente para este estudo. Para ser incluído, o paciente tinha que cumprir o critério de acidente vascular cerebral e tinha que apresentar comprometimento cognitivo ${ }^{55}$.

O primeiro destes dois experimentos visava demonstrar se um aumento de potência em alfa, através de neurofeedback, poderia ter efeitos positivos na restauração do desempenho cognitivo pós-AVC ${ }^{55}$. Tendo como base publicações que afirmam, que uma maior amplitude de alfa alta, ou seja, um pico de amplitude das ondas alfa na faixa de $10-12 \mathrm{~Hz}$ está relacionado à melhora do desempenho cognitivo ${ }^{50,56}$.

O segundo experimento visava verificar se as deficiências cognitivas relacionadas ao pós-AVC eram devido á grande amplitude na faixa teta ou à baixa amplitude na faixa alfa ${ }^{55}$. Baseando-se tanto na afirmação de que uma maior amplitude de alfa alta, quanto uma menor amplitude na faixa teta, ou seja, um aumento da quantidade de energia durante a tarefa está associado com bom desempenho cognitivo ${ }^{49,57}$.

O experimento $\mathrm{n}^{\mathrm{o}} 1$ foi feito com trinta e dois participantes com idade média de 56 anos, os quais foram divididos em um grupo controle com dezessete participantes e um grupo experimental com quinze participantes. Todos os participantes receberam treinamento informatizado, sendo que o grupo controle foi submetido a um treino de relaxamento com biofeedback e o grupo experimental foi submetido ao neurofeedback com a finalidade de aumentar a potência em alfa ${ }^{55}$.

Para testar a hipótese do segundo experimento, foram comparados três diferentes protocolos de treinamento: (1) redução do poder de teta, (2) aumento da potência em alfa e (3) como uma condiçáo de controle, treinamento de biofeedback da arritmia sinusal respiratória (ASR). Neste experimento $\mathrm{n}^{\circ} 2$, o grupo de neurofeedback teta foi composto de quatro participantes, o grupo de neurofeedback alfa de sete participantes, e o grupo ASR de seis participantes. Sendo que oito sessóes de treinamento foram realizadas por cada paciente ${ }^{55}$. 
Os dados do experimento $\mathrm{n}^{\mathrm{o}} 1$ foram promissores e claramente demonstraram um aumento significativo apenas no desempenho do grupo alfa. Não houve avanço para o grupo de relaxamento. Então, o neurofeedback alfa mostrou ser uma valiosa ferramenta para a reabilitação cognitiva, pois o resultado foi superior ao do grupo controle, que recebia a mesma quantidade de apoio emocional, ou outras variáveis relacionadas a um potencial efeito placebo ${ }^{55}$.

No entanto, o experimento 2, não revelou provas concisas para a eficácia do neurofeedback. Todos os três grupos obtiveram melhora equivalente no desempenho cognitivo. Nem o neurofeedback alfa e nem o teta apresentaram resultados superiores ao grupo controle de treinamento com biofeedback da ASR ${ }^{55}$.

Nos dois experimentos, os resultados diferiram consideravelmente. Contrapondo-se às consideraçóes teóricas e aos resultados de estudos anteriores ${ }^{50}$, nos quais se assumia que o neurofeedback poderia apoiar o restabelecimento do desempenho cognitivo após um AVC. Uma explicação plausível, para este resultado do experimento $\mathrm{n}^{\circ} 2$, poderia ser a de que o programa de reabilitação padrão da clínica foi eficiente em todos os grupos e não o treinamento específico de cada grupo. Neste caso, nenhum dos três diferentes protocolos de treinamento seria eficiente. Outra explicação possível seria que o treinamento com feedback no computador foi eficaz, mas todos os três treinos tinham efeitos semelhantes ${ }^{55}$.

Baseando-se no modelo conceitual, os autores do estudo esperavam que o desempenho cognitivo aumentasse com o neurofeedback teta e com o neurofeedback alfa, mas não se esperava um aumento comparável com o biofeedback ASR, pois não havia, até o momento, qualquer referencial teórico claro que poderia explicar esse benefício cognitivo com o treinamento da ASR ${ }^{55}$.

Além desses resultados contraditórios, devem-se reconhecer várias falhas metodológicas em ambos os estudos. A primeira deficiência é o pequeno tamanho da amostra, em especial no grupo de neurofeedback teta. Além disso, teria sido favorável comparar estes dados com dois outros grupos: com um grupo sem nenhuma intervenção e com outro grupo de pacientes que recebessem apenas o programa de reabilitação padrão da clínica ${ }^{55}$.

Analisando os resultados destes dois experimentos, realizados na Clínica de Reabilitação Medical Park Loipl, pode-se afirmar que o uso do neurofeedback apresentou melhorias significativas apenas no primeiro experimento. Por causa de suas limitações metodológicas, ainda não se pode tirar conclusões confiáveis sobre a eficácia do neurofeedback para a restauração cognitiva pós-AVC, baseadas nestes dois estudos. No entanto, o neurofeedback parece promissor, devendo-se concentrar estudos futuros sobre este tipo de reabilitação com metodologia mais sofisticada.

\section{CONCLUSÃO}

O EEG biofeedback ou neurofeedback se apresenta como um novo tratamento para disfunçóes cognitivas e psicológicas decorrentes de acidente vascular cerebral, inclusive fornecendo benefícios para pacientes fora do intervalo de tempo em que é esperada uma melhora espontânea.

Em relação à reabilitação cognitiva, o campo do neurofeedback, está se configurando como uma grande promessa para a reabilitação da atenção, processamento da linguagem e memória de trabalho. Em relação à reabilitaçâo psicológica, o neurofeedback vem apresentando, como principais efeitos clínicos, uma diminuição da depressão e da impulsividade e uma maior estabilidade de humor.

O neurofeedback tem uma utilidade clínica única, que consiste em demonstrar aos pacientes, que eles podem exercer controle voluntário sobre alguns aspectos do seu funcionamento, que anteriormente eles faziam de forma não consciente. Esta demonstração é um argumento para a inclusão desta intervenção, o mais cedo possível, na reabilitação pós-AVC, potencializando a esperada recuperação espontânea.

Embora o neurofeedback demonstre ser efetivo no tratamento de diferentes manifestaçôes clínicas, inclusive no traumatismo craniano e no acidente vascular cerebral, ele é subutilizado na clínica brasileira. O que pode ocorrer por várias razões, algumas delas podem ser equipamentos e formação de alto custo. No entanto, a principal razão pode ser simplesmente a falta de conhecimento, por parte de alguns profissionais, sobre as pesquisas científicas e sobre o potencial de eficácia dessa abordagem na reabilitação pós-AVC. 
Entretanto, é fundamental a elaboração de estudos para investigar algumas questóes: a intervenção com neurofeedback, o mais cedo possível após a lesão, diminuiria a severidade das sequelas? Será que o uso do neurofeedback combinado com outra terapia convencional aumentaria a velocidade de recuperação? Será o neurofeedback capaz de promover a recuperação funcional em pacientes que sofreram AVC há muitos anos? Qual protocolo de treinamento do neurofeedback, dependendo do tipo de lesão, tem um melhor resultado clínico?

O neurofeedback deve ser considerado como uma alternativa na reabilitação neuropsicológica pós-AVC por ser um procedimento não invasivo, livre de efeitos colaterais e que vem mostrando resultados positivos nos trabalhos $^{32,36,52-55}$. No entanto, são necessários mais estudos para demonstrar as evidências experimentais de sua eficácia, o que não impede seu uso na prática clínica.

\section{REFERÊNCIAS}

1.O’Sullivan SB, Schmitz TJ. Fisioterapia: Avaliação e Tratamento. 2a ed. São Paulo: Manole, 1993, 775p.

2.Centers for Disease Control And Prevention. Disparities in deaths from stroke among persons aged $<75$ years - United States, 2002. MMWR Morb Mortal Wkly Rep 2002;54:477-81.

3.Centers for Disease Control And Prevention. Prevalence of disabilities and associated health conditions-United States, 1991-92. MMWR Morb Mortal Wkly Rep 1994;43:730-9.

4.American Heart Association. Heart disease and Stroke Statistics -2000 Update. Dallas: American Heart Association; 1999, 37p.

5.Page SJ, Gater DR, Bach-Y-Rita P. Reconsidering the motor recovery plateau in stroke rehabilitation. Archives of Physical Medicine and Rehabilitation 2004;85:1377-81.

http://dx.doi.org/10.1016/j.apmr.2003.12.031

6.Paolucci S, Antonucci G, Grasso MG, Bragoni M, Coiro P, De Angelis D, et al. Functional outcome of ischemic and hemorrhagic stroke patients after inpatient rehabilitation: a matched comparison. Stroke 2003;34:2861-5.

http://dx.doi.org/10.1161/01.STR.0000102902.39759.D3

7.Erkinjuntti T, Roman G, Gauthier S, Feldman H, Rockwood K. Emerging therapies for vascular dementia and vascular cognitive impairment. Stroke 2004;35:1010-7.

http://dx.doi.org/10.1161/01.STR.0000120731.88236.33

8.Duncan PW, Horner RD, Reker DM, Samsa GP, Hoenig H, Hamilton B, et al. Adherence to post acute rehabilitation guidelines is associated with functional recovery in stroke [editorial comment]. Stroke 2002;33:167-78.

http://dx.doi.org/10.1161/hs0102.101014

9.Van Peppen RPS KG, Wood-Dauphinee S, Hendriks HJM, Van der Wees PhJ, Dekker J. The impact of physical therapy on functional outcomes after stroke: What's the evidence? Clin Rehabil 2004;18:833-62. http://dx.doi.org/10.1191/0269215504cr843oa

10.Walker MF, Leonardi-Bee J, Bath P, Langhorne P, Dewey M, Corr S, et al. Individual patient data meta-analysis of randomized controlled trials of community occupational therapy for stroke patients. Stroke 2004;35:2226-32. http://dx.doi.org/10.1161/01.STR.0000137766.17092.fb

11.Cicerone KD, Dahlberg C, Malec JF, Langenbahn DM, Felicetti T, Kneipp $S$, et al. Evidence-based cognitive rehabilitation: updated review of the literature from 1998 through 2002. Arch Phys Med Rehabil 2005;86:1681-92.

http://dx.doi.org/10.1016/j.apmr.2005.03.024

12.Levin HS. Cognitive rehabilitation: Unproved but promising. Archives of Neurology. 1990;47:223-4.

http://dx.doi.org/10.1001/archneur.1990.00530020131027

13.Brosseau L, Wells GA, Finestone HM, Egan M, Dubouloz CJ, McGowan $\mathrm{J}$, et al. Ottawa panel evidence-based guidelines for post-stroke rehabilitation. Top Stroke Rehabil 2006;13:1-269.

http://dx.doi.org/10.1310/3TKX-7XEC-2DTG-XQKH

14.Monastra VJ, Monastra DM, George S. The effects of stimulant therapy, EEG biofeedback, and parenting style on the primary symptoms of attentiondeficit/hyperactivity disorder. Applied Psychophysiology and Biofeedback 2002;27:231-49.

http://dx.doi.org/10.1023/A:1021018700609

15.Evans JR, Abarbanel A. Introduction to Quantitative EEG and Neurofeedback. New York: Academic Press; 1999, 406p.

16.Alper KR, John ER, Brodie J, Gunther W, Daruwala R, Prichep LS. Correlation of PET and QEEG in normal subjects. Psychiatry Res: Neuroimaging 2006;146:271-82.

http://dx.doi.org/10.1016/j.pscychresns.2005.06.008

17.Lee JS, Lee DS, Oh SH, Kim CS, Kim JW, Hwang CH, et al. PET evidence of neuroplasticity in adult auditory cortex of post lingual deafness. J Nucl Med 2003;44:1435-9.

18.Detre JA. Clinical applicability of functional MRI. J Magn Reson Imaging 2006;23:808-15.

http://dx.doi.org/10.1002/jmri.20585

19. Crane A, Soutar R. Mind fitness Training: Neurofeedback and the Process. San Jose: Writers Club Press; 2000, 408p.

20.Thompson M, Thompson L. The Neurofeedback Book. Whear Ridge: The Association for Applied Psychophysiology and Biofeedback; 2003, 457p.

21.Sterman MB, Egner T. Foundation and practice of neurofeedback for the treatment of epilepsy. Appl Psychophys Biofeedback 2006;31:21-35.

http://dx.doi.org/10.1007/s10484-006-9002-x

22.Strehl U, Trevorrow T, Veit R, Hinterberger T, Kotchoubey B, Erb M, et al. Deactivation of brain areas during selfregulation of slow cortical potentials in seizure patients. Appl Psychophys Biofeedback 2006;31:85-94.

http://dx.doi.org/10.1007/s10484-006-9006-6

23.Kaiser DA, Othmer S. Effect of neurofeedback on variables of attention in a large multi-center trial. J Neurotherapy 2000;4:5-15.

http://dx.doi.org/10.1300/J184v04n01_02

24.Lubar J F, Lubar JO. Neurofeedback assessment and treatment for attention deficit/hyperactivity disorders. In J. R. Evans, A. Abarbanel (Eds.). Introduction to quantitative EEG and neurofeedback. San Diego: Academic Press; 1999, p.103-43.

http://dx.doi.org/10.1016/B978-012243790-8/50006-7

25.Thompson L, Thompson M. Neurofeedback combined with training in metacognitive strategies: Effectiveness in students with ADD. Appl Psychophys Biofeedback 1998;23:243-63.

http://dx.doi.org/10.1023/A:1022213731956

26.Othmer S, Othmer SF, Kaiser D. EEG Biofeedback: A Generalized Approach to Neuroregulation Section 2 presented within a course manual; EEG Bio- 
feedback Certification student binder \#1, academic year 2000/2001. Phoenix: Neurofeedback Performance Institute; 2001; 13p.

27.Peniston EG, Kulkosky PJ. Alcoholic personality and alpha-theta brainwave training. Med Psychother 1990;3:37-55.

28. Michel JA, Mateer CA. Attention rehabilitation following stroke and traumatic brain injury: a review. Eur Med Phys 2006;42:59-67.

29.Robbins J. The mental edge. Outside Magazine 2001;XXVI(4):131-4.

30.Egner T, Gruzelier JH. Ecological validity of neurofeedback: Modulation of slow wave EEG enhances musical performance. NeuroRepor 2003;14:1221-4. http://dx.doi.org/10.1097/00001756-200307010-00006

31.Vernon D, Egner T, Cooper N, Compton T, Neilands C, Sheri A, et al. The effect of distinct neurofeedback protocols on aspects of cognitive performance. Intl J Psychophys 2003;47:75-85.

http://dx.doi.org/10.1016/S0167-8760(02)00091-0

32.Ayers ME. A report on a study of the utilization of electro-encephalography (Neuroanalyzer) for the treatment of cerebral vascular lesion syndromes. In: Taylor LP, Ayers ME, Tom G, eds. Electromyometric Biofeedback Therapy. Los Angeles: Biofeedback and Advanced Therapy Institute; 1981:244-57.

33.Ayers M. Assessing and treating open head trauma, coma, and stroke using real-time digital EEG neurofeedback. In J. R. Evans, A. Abarbanel (Eds.). Introduction to quantitative EEG and neurofeedback. San Diego: Academic

Press, 1999; p.203-22.

http://dx.doi.org/10.1016/B978-012243790-8/50010-9

34.Catani M, Ffytche DH. The rises and falls of disconnection syndromes. Brain 2005;128:2224-39.

http://dx.doi.org/10.1093/brain/awh622

35.Walker JE, Kozlowski GP, Lawson R. A modular activation/coherence approach to evaluating clinical/QEEG correlations and for guiding neurofeedback training: Modular insufficiencies, modular excesses, disconnections, and hyperconnections. J Neurotherapy. 2007;11:25-44.

http://dx.doi.org/10.1300/J184v11n01 03

36.Ayers ME. Electroencephalic Neurofeedback and Closed Head Injury of 250 Individuals. Los Angeles: Neuropathways EEG Imaging; 1987, 380p.

37.Geschwind N. Disconnexion syndromes in animals and man. I. Brain 1965;88:237-94.

http://dx.doi.org/10.1093/brain/88.2.237

http://dx.doi.org/10.1093/brain/88.3.585

38.Geschwind N. Disconnexion syndromes in animals and man. II. Brain1965;88:585-644.

http://dx.doi.org/10.1093/brain/88.2.237

http://dx.doi.org/10.1093/brain/88.3.585

39.Walker JE. A neurologist's experience with QEEG-guided neurofeedback following brain injury. In J. R. Evans (Ed.). Handbook of neurofeedback. Binghamton: Haworth Press; 2007, p.353-61.

40.Walker JE, Norman CA. The neurophysiology of dyslexia: A selective review with implications for neurofeedback remediation and results of treatment in twelve consecutive patients. J Neurotherapy 2006;10:45-55.

http://dx.doi.org/10.1300/J184v10n01_04

41.Walker JE, Kozlowski GP. Neurofeedback treatment of epilepsy. Child Adolesc Psychiatric Clin N Am 2005;14:163-76.

http://dx.doi.org/10.1016/j.chc.2004.07.009

42.Coben R. Connectivity-guided neurofeedback for autistic spectrum disorder. Biofeedback 2007;35:131-5.
43. Courchesne E, Pierce K. Why the frontal cortex in autism might be talking only to itself: Local overconnectivity, but longdistance disconnection. Curr Opin Neurobiol 2005;15:225-30.

http://dx.doi.org/10.1016/j.conb.2005.03.001

44.Harper SH. In support of neuroplasticity: All digital real time EEG biofeedback with coma. Abstracts of general conference presentations at the 2003 International Society of Neurofeedback and Research 11 th annual conference, Houston, TX. J Neurotherapy 2005;8:85-132.

45.Thornton KE, Carmody DP. Efficacy of traumatic brain injury rehabilitation: Interventions of QEEG-guided biofeedback, computers, strategies and medication. Appl Psychophys Biofeedback 2008;33:101-24.

http://dx.doi.org/10.1007/s10484-008-9056-z

46.Hammond DC. Can LENS neurofeedback treat anosmia resulting froma head injury? J Neurotherapy 2007;11:57-62.

http://dx.doi.org/10.1300/J184v11n01_06

47.Larsen S. The healing power of neurofeedback. Rochester: Healing Arts; 2006, 432p.

48.Larsen S, Esty ML, Ochs L. Traumatic brain injury (TBI) and spinal injury and the LENS. In The healing power of neurofeedback. Rochester: Healing Arts; 2006, p.97-124.

49.Klimesch W. EEG alpha and theta oscillations reflect cognitive and memory performance: A review and analysis. Brain Res Revs 1999;29:169-95.

http://dx.doi.org/10.1016/S0165-0173(98)00056-3

50.Hanslmayr S, Sauseng P, Doppelmayr M, Schabus M, Klimesch W. Increasing individual upper alpha power by neurofeedback improves cognitive performance in healthy subjects. Appl Psychophys Biofeedback 2005;30:1-10. http://dx.doi.org/10.1007/s10484-005-2169-8

51.Byers AP. Neurofeedback therapy for a mild head injury. J Neurother 1995; 1:22-37.

http://dx.doi.org/10.1300/J184v01n01_04

52.Rozelle GR, Budzynski TH. Neurotherapy for stroke rehabilitation: A single case study. Biofeedback Self Regul 1995;20:211-28.

http://dx.doi.org/10.1007/BF01474514

53.Laibow RE, Stubblebine AN, Sandground H, Bounias M. EEG-neurobiofeedback treatment of patients with brain injury: Part 2: Changes in EEG parameters versus rehabilitation. J Neurother 2001;5:45-71.

http://dx.doi.org/10.1300/J184v05n04 04

54.Bearden TS, Cassisi JE, Pineda M. Neurofeedback training for a patient with thalamic and cortical infarctions. Appl Psychophysiol Biofeedback 2003;28:241-53.

http://dx.doi.org/10.1023/A:1024689315563

55.Doppelmayr M, Nosko H, Pecherstorfer T, Fink A. An Attempt to Increase Cognitive Performance After Stroke With Neurofeedback. Biofeedback 2007;35:126-30.

56.Klimesch W, Sauseng P, Hanslmayr S. EEG alpha oscillations: The inhibition-timing hypothesis. Brain Res Revs 2006;53:63-88.

http://dx.doi.org/10.1016/j.brainresrev.2006.06.003

57.Doppelmayr M, Klimesch W, Hödlmoser K, Sauseng P, Gruber W. Intelligence related upper alpha desynchronisation in a semantic memory task. Brain Res Bull 2005;66:171-7.

http://dx.doi.org/10.1016/j.brainresbull.2005.04.007

58.Rowan AJ, Tolunsky E. Primer of EEG: with a Mini-Atlas. Philadelphia: Elsevier; 2003, 224p. 